Sportwiss 2009 · 39:262-262

DOI 10.1007/s12662-009-0060-0

Online publiziert: 20. August 2009

๑) Springer Medizin Verlag 2009
Yvonne Steggemann

Neurocognition and Action - Biomechanics Research Group,

Faculty of Psychology and Sport Sciences, Bielefeld University, Bielefeld

\title{
Sportwissenschaft erstmals mit einem Spezialkurs vertreten
}

\author{
Interdisziplinäres Kolleg (IK) vom \\ 6. bis 13. März 2009, Günne am Möhnesee
}

Die Organisatoren boten in diesem Jahr unter dem Schwerpunktthema „Rhythm and Timing" auch aus Sicht der Sportwissenschaft ein vielfältiges und interessantes Kursangebot. Das Programm setzte sich aus Basis-, Methoden- und Spezialkursen zusammen, die einerseits Einführungen in die Kerngebiete des IK gaben und andererseits das Fokusthema aus den verschiedenen Disziplinen beleuchteten (weitere Informationen unter www.ik2oog. de). Darüber hinaus wurde das Programm durch Posterbeiträge der Teilnehmer/innen, Abendvorträge und Diskussionsrunden ergänzt. Viele weitere (in-) offizielle Aktivitäten und ein zusätzlicher Praxiskurs sorgten für den nötigen Ausgleich und gleichzeitig für eine praktische Beschäftigung mit dem Schwerpunktthema.

Erstmalig war auch die Sportwissenschaft mit einer eigenen Veranstaltung im Kursprogramm durch Matthias Weigelt von der Universität Bielefeld vertreten. Sein Spezialkurs zum Thema „Motor Coordination Learning during Childhood and Adolescence" fügte sich hervorragend ins Schwerpunktthema ein und traf auf eine interessierte Zuhörerschaft. Über insgesamt vier Sitzungen hinweg wurden die Grundlagen der motorischen Entwicklung und des Erwerbs komplexer (sportlicher) Fertigkeiten im Kleinkind- und Schulalter vermittelt. Für dieses Thema interessierten sich nicht nur die Kolleginnen und Kollegen aus der Sportwissenschaft, die seit einigen Jahren am IK teilnehmen. Besonders zahlreich vertreten waren diesmal die Arbeitsgruppen aus Bielefeld und Gießen, die ihre aktuellen Forschungsprojekte im Rahmen der beiden Postersessions vorstellten. In Zukunft sollten sich noch mehr Kolleginnen und Kollegen von diesem fachübergreifenden Kursangebot angesprochen fühlen. Denn nicht nur das offizielle Programm macht das IK zu einer einzigartigen Veranstaltung, sondern auch die familiäre Atmosphäre der kleinen Diskussionsrunden und die persönlichen Kontakte, welche in diesem Rahmen entstehen.

\section{Korrespondenzadresse \\ Yvonne Steggemann \\ Sportwissenschaftlerin (M.A.) \\ Neurocognition and Action - \\ Biomechanics Research Group, \\ Faculty of Psychology and Sport Sciences, \\ Bielefeld University \\ Universitätsstr. 25, 33615 Bielefeld \\ yvonne.steggemann@uni-bielefeld.de}

Interessenkonflikt. Die korrespondierende Autorin gibt an, dass kein Interessenkonflikt besteht. 